\title{
Implementación de un Laboratorio de Física en Tiempo Real para el Aprendizaje Activo de Circuitos Eléctricos
}

\author{
Federico G. Rosales, Viviana M. Mercado, Ricardo R. Monasterolo y Sergio L. Ribotta \\ Facultad de Ingeniería y Ciencias Agropecuarias, Universidad Nacional de San Luis, Avenida 25 de Mayo \\ №384, (D5730EKQ) Villa Mercedes, San Luis, Argentina (e-mail: fgrosale@gmail.com; \\ mercadocamiolo@yahoo.com.ar; ricardomonasterolo@gmail.com; sergioribotta@gmail.com)
}

Recibido Mar. 4, 2016; Aceptado Abr. 11, 2016; Versión final Jun. 9, 2016, Publicado Dic. 2016

\begin{abstract}
Resumen
El objetivo del presente estudio es analizar el uso de un laboratorio para la enseñanza de Circuitos Eléctricos utilizando la metodología de enseñanza Física en Tiempo Real (RTP, Real Time Physics). La experiencia se realizó con alumnos de Física en carreras de Ingeniería de la Universidad Nacional de San Luis, en Argentina. Se formaron dos grupos: un grupo control instruido mediante enseñanza tradicional y otro grupo experimental, instruido a través de los laboratorios RTP. Como instrumento de medición se utilizó un test de respuestas múltiples que contiene una gran variedad de concepciones alternativas y dificultades de aprendizaje sobre circuitos eléctricos. Los resultados experimentales sobre el rendimiento de los alumnos muestran que se logró una mejora en el aprendizaje conceptual respecto a la enseñanza tradicional.
\end{abstract}

\section{Implementing a Real Time Physics Laboratory (RTP) for the Active-Learning of Electric Circuits}

\begin{abstract}
The purpose of this project is to analize the use of a laboratory to teach electric circuits by means of Real Time Physcis (RTP). The research was carried out with students of Physics studying Engineering at the National University of San Luis, in Argentina. For the experiment, the students were divided into two groups: a control group that received traditional instruction and an experimental group that received instruction through RTP laboratories. A test of multiple responses, which contains a wide variety of alternative conceptions and difficulties to learn about electric circuits, was used to measure the results. The experimental results show that improvement of conceptual learning over traditional learning was achieved.
\end{abstract}




\section{INTRODUCCIÓN}

La investigación educativa de los últimos 20 años ha demostrado que estudiantes sometidos a una instrucción tradicional (aquella basada en clases expositivas, resolución de problemas algorítmicos y laboratorios tipo receta) mantienen un aprendizaje residual casi nulo (Benegas, 2009). Según RegaladoMendez (2014), "el método tradicional no estimula a los estudiantes a tener una participación activa en su aprendizaje, profundidad en el entendimiento de los conceptos fundamentales, en la aplicación de dichos conceptos para la resolución activa de problemas y mucho menos en el diseño de nuevos productos". Por otro lado, este método tradicional de enseñanza no tiene en cuenta los conocimientos adquiridos con anterioridad (preconcepciones), los cuales, influyen negativamente en el aprendizaje de temas cruciales (Ausubel et al., 1976).

Es por esto que los investigadores en este tema han comenzado a abordar este problema desde una perspectiva científica mediante la realización de estudios sistemáticos detallados sobre el aprendizaje y la enseñanza de la física. Con esta base científica de las dificultades de aprendizaje y los modelos alternativos más relevantes aportados por la investigación educativa, algunos grupos de investigación han desarrollado metodologías de enseñanza destinadas a favorecer el aprendizaje conceptual y significativo de la física en alumnos de nivel secundario y universitario (McDermott y Redish, 1999). Una de estas estrategias didácticas, basada en el uso de Laboratorios, es Real Time Physics (RTP) (Sokoloff et al., 2012) desarrollados por el grupo de investigación en enseñanza de la física liderado por el Prof. David Sokoloff en la University of Oregon, USA.

Para los docentes de nuestra área un trabajo importante es evaluar objetivamente los resultados de aprendizaje de sus propias clases, de manera tal que puedan ser comparados con cursos anteriores. El objetivo en esta investigación es poder obtener datos cuantificables para realizar la comparación efectiva entre una clase sometida a instrucción tradicional y otra clase que utiliza una metodología basada en la enseñanza activa de física, en este caso la metodología RTP. Como se menciona anteriormente, esta metodología tiene como fin favorecer el aprendizaje conceptual y significativo de la física, está pensada para ser implementada como laboratorio y puede ser utilizada complementariamente con otras actividades. RTP utiliza diferentes recursos para lograr que los alumnos pongan en discusión sus conocimientos sobre el tema en particular de circuitos eléctricos, comparando sus predicciones (ideas previas) con la realidad que brinda el experimento.

Considerando la concepción de aprendizaje, las teorías constructivistas sostienen que las personas aprenden interactuando con los objetos y con otros sujetos en el marco de una cultura, en esa interacción los sujetos modifican la realidad a la vez que se modifican los esquemas del sujeto, siendo estos esquemas representaciones de una situación concreta o de un concepto que permite manejarlos internamente y enfrentarnos a situaciones iguales o parecidas a la realidad (Castorina, 1998). En este proceso de interacción con el objeto de conocimiento Ausubel (1976) concibe el aprendizaje, como significativo, cuando existe un proceso de adquisición de significados a través del establecimiento de relaciones sustantivas entre el conocimiento previo y el nuevo material de aprendizaje. Es por ello, que considera a la exposición organizada de contenidos como un instrumento muy eficaz para conseguir una comprensión adecuada por parte de los alumnos. En este sentido, sugiere seleccionar y estructurar los contenidos de modo de favorecer la comprensión, teniendo en cuenta, no solo el punto de vista de la disciplina, sino considerando el nivel de dificultad que le ofrece al alumno. Por todo esto, contar con una estrategia de enseñanza que tenga en cuenta los conocimientos previos, las diferentes capacidades de los alumnos y que logre una comprensión del tema planteado constituye una metodología que aporta a la construcción del conocimiento.

Se puede establecer entonces que el Aprendizaje Activo de la Física es un conjunto de estrategias y metodologías para la enseñanza de la Física (Benitez y Mora, 2010), en donde "los alumnos son guiados a construir su conocimiento mediante observaciones directas del mundo físico" (Mora, 2008). Para poder establecer las diferencias que existen entre el aprendizaje pasivo o tradicional y el aprendizaje activo se muestra la siguiente tabla comparativa (Tabla 1) (Benitez y Mora, 2010).

\section{DISEÑO DE INVESTIGACIÓN}

Se propone en esta investigación un estudio de comparación de grupos con Pre-test y Post-test. El diseño se encuadra dentro lo que se denomina, según Crowl (2002), un cuasi-experimento. La población de aplicación corresponde a alumnos de las carreras de Ingeniería Electrónica, Electromecánica, Industrial, Química y Alimentos. Las muestras tratadas en este trabajo son parte de un grupo existente antes de comenzar el estudio y que está conformado por los alumnos que cursan la asignatura Física II de la Facultad de Ingeniería y Ciencias Agropecuarias (FICA) de la Universidad Nacional de San Luis (UNSL). 
Tabla 1: Ambientes de aprendizaje pasivo vs aprendizaje activo

\begin{tabular}{|l|l|}
\hline \multicolumn{1}{|c|}{ Aprendizaje Pasivo } & \multicolumn{1}{|c|}{ Aprendizaje Activo } \\
\hline Transmitir contenidos & Enseñar a aprender \\
\hline Formación técnica & Formación integral \\
\hline $\begin{array}{l}\text { El profesor y/o los libros son la autoridad y la única } \\
\text { fuente de conocimiento }\end{array}$ & $\begin{array}{l}\text { El profesor y/o los libros son una guía en el proceso de } \\
\text { aprendizaje. Las observaciones del mundo físico son la } \\
\text { autoridad }\end{array}$ \\
\hline $\begin{array}{l}\text { Las concepciones de los estudiantes son raramente } \\
\text { analizadas y comprendidas }\end{array}$ & $\begin{array}{l}\text { El aprendizaje activo de la física permite el cambio } \\
\text { conceptual a través del compromiso y la participación }\end{array}$ \\
\hline $\begin{array}{l}\text { Los estudiantes no reconocen la diferencia entre sus } \\
\text { concepciones y lo que se dijo en clase }\end{array}$ & $\begin{array}{l}\text { Los estudiantes reconocen las diferencias entre sus } \\
\text { ideas previas y lo observado }\end{array}$ \\
\hline $\begin{array}{l}\text { El profesor construye el conocimiento del alumno, } \\
\text { asume la responsabilidad del aprendizaje }\end{array}$ & $\begin{array}{l}\text { Los estudiantes construyen su propio conocimiento y } \\
\text { asumen la responsabilidad de su aprendizaje }\end{array}$ \\
\hline $\begin{array}{l}\text { No es posible el trabajo colaborativo } \\
\text { El trabajo de laboratorio se utiliza para confirmar las } \\
\text { teorías dadas }\end{array}$ & $\begin{array}{l}\text { El trabajo colaborativo permite el análisis conceptual de } \\
\text { forma cuidadosa }\end{array}$ \\
\hline
\end{tabular}

Los grupos a comparar fueron asignados a partir de estudiantes de una clase pre-establecida, los cuales son instruidos según distintos métodos de enseñanza. Es por esto que nuestra búsqueda se concentra en encontrar la diferencia en puntajes promedio con respecto a una misma variable en dos grupos de alumnos. Por lo tanto ambos grupos difieren en términos del método de enseñanza recibido, es decir, un grupo recibe el Método $\mathrm{A}$ y el otro el Método B. El método de enseñanza se convierte en la variable, y los dos tipos de enseñanza son los valores que la variable puede asumir en nuestro caso (Campbell, 1963).

Como se mencionó anteriormente este trabajo se encuentra dentro de lo que se denomina un cuasiexperimento, que según la definición de Bono Cabré, "un cuasi experimento es un conjunto de procedimientos o estrategias de investigación orientado a la evaluación del impacto de los tratamientos en aquellos contextos donde la asignación de las unidades no es al azar, y al estudio de los cambios que se observan en los sujetos en función del tiempo" (Bono, 1997). Según expone Crowl en los diseños cuasiexperimentales el conjunto de sujetos no se asignan de manera aleatoria sino que ya estaban formados antes del experimento, lo que él llama grupos o clases intactas. Como se muestra más adelante, como no existe una aleatorización de los sujetos que van a conformar los grupos, Crowl sugiere que se verifique la equivalencia de los mismos utilizando las medias y desviaciones estándar del Pre-test (Crowl, 2002).

Debido a los distintos requisitos impuestos por la institución, los planes de estudio de las distintas carreras y la asignatura, como por ejemplo exámenes de ingreso, cursado de materias correlativas (Matemática, Algebra y Geometría Analítica, Análisis Matemático y Física I), podemos establecer que los grupos conformados para realizar el estudio están en igualdad de condiciones al comenzar la asignatura. De todas maneras para corroborar que los grupos resulten equivalentes para un análisis estadístico se administra al principio un examen pre-test denominado DIRECT que nos permite determinar si los grupos asignados a distintos valores de la variable independiente (a saber, método de enseñanza) conforman grupos equivalentes al comenzar la investigación.

Como se mencionó esta investigación de comparación de grupos responde a un diseño que se observa en la Tabla 1, donde a partir de dos comisiones formadas de la clase original se asigna una para que siga el método de Enseñanza Tradicional (Grupo Control), mientras que la otra recibe el método de enseñanza experimental (Grupo Experimental). Todos los estudiantes, como se mencionó, responden al cuestionario dos veces: una como Pre-test (O1) antes de la instrucción $(X)$ y nuevamente como Post-test (O2) al finalizar la instrucción. Según expone Benegas y Zavala (2013) este método proporciona la información más completa, ya que por un lado utilizando los resultados del Pre-test, se puede obtener si los grupos resultan equivalentes al comenzar la instrucción, y por otro lado, se puede analizar en qué temas o aspectos la instrucción fue más o menos efectiva.

Tabla 2: Diseño de Investigación

\begin{tabular}{|l|c|c|c|}
\hline & Pre-test & Tratamiento & Post-test \\
\hline Grupo Experimental & $\mathrm{O}_{1}$ & $\mathrm{X}$ & $\mathrm{O}_{2}$ \\
\hline Grupo Control & $\mathrm{O}_{1}$ & & $\mathrm{O}_{2}$ \\
\hline
\end{tabular}


De acuerdo al análisis que se realiza de los datos obtenidos experimentalmente, este trabajo se enmarca dentro de lo que se denomina una investigación cuantitativa. Este tipo de estudio es aquel que permite examinar los datos de manera numérica. Estos datos experimentales, al ser interpretados numéricamente, informan si la intervención realizada en determinadas situaciones tiene influencias positivas (Calero, 2000). Es por esto que en nuestro caso en particular se justifica la utilización de una investigación del tipo cuantitativa donde se analizan diversos elementos que pueden ser medidos y cuantificados.

La presente investigación se sirve de números y métodos estadísticos, parte de un caso concreto para lograr llegar a una descripción general o comprobar hipótesis causales (Campbell y Stanley, 2002). Se plantean los siguientes objetivos centrales: (i) Experimentar con muestras estudiantiles representativas la aplicación de la estrategia RTP y su adaptación a las necesidades locales; (ii) Evaluar el impacto de esta estrategia en el aprendizaje conceptual de circuitos eléctricos, comparado con la enseñanza tradicional; y (iii) Mostrar el uso de un test de respuestas múltiples diseñado científicamente para evaluar el aprendizaje conceptual sobre circuitos eléctricos simples.

\section{DESCRIPCIÓN DE LA MUESTRA}

El estudio se llevó a cabo en la FICA, más específicamente en el área de Física donde se dicta la asignatura Física II para las carreras de Ingeniería mencionadas anteriormente. Luego de recibir a los alumnos que cumplen con las condiciones mínimas establecidas por la institución para cursar la asignatura se procede a conformar los grupos en particular. Con el objetivo de lograr una equivalencia de estos grupos se utilizan los resultados de las respuestas correctas por individuo del Pre-test, se agrupan a los estudiantes de tal manera de que el promedio de aciertos en sus respuestas sea similar entre todas las comisiones, una vez realizado esto los grupos quedaron conformados según se observa en la Tabla 3: Grupo control, denominado "Control_A", es aquel que recibirá instrucción tradicional. Como grupos experimentales, los cuales son sometidos a la estrategia en estudio, se consideran dos: el restante de alumnos del mismo año que el grupo control, "Experimental_A", y el otro grupo, "Experimental_B", a la totalidad de alumnos del año posterior. Cabe aclarar que este último grupo experimental se consideró a fines de poder realizar una comparación entre un año de cursado y el siguiente.

Tabla 3: Características de la muestra

\begin{tabular}{|c|c|c|c|c|c|c|}
\hline Muestra & $\begin{array}{c}\text { Universidad } \\
\text { Facultad }\end{array}$ & Año & Carrera & Género & Edad & № Alumnos \\
\hline Control_A & \multirow{3}{*}{$\begin{array}{l}\text { UNSL } \\
\text { FICA }\end{array}$} & \multirow{3}{*}{$2^{\circ}$ año } & \multirow{3}{*}{ Ingenierías } & \multirow{3}{*}{ Mixto } & \multirow{3}{*}{$19-20$} & 20 \\
\hline Experimental_A & & & & & & 31 \\
\hline Experimental_B & & & & & & 27 \\
\hline
\end{tabular}

Salvo por diferencias ajenas al experimento las condiciones de instrucción fueron similares para las tres comisiones, donde todas tienen el mismo profesor responsable y la misma carga horaria de dictado de clases teóricas y prácticas con resolución de problemas.

\section{METODOLOGÍAS DE ENSEÑANZA EXPERIMENTAL Y TRADICIONAL}

Se describe el concepto de física en tiempo real y luego se explica y analiza la instrucción tradicional grupo control.

\section{Física en Tiempo Real (Real Time Physics, RTP)}

Este tipo de laboratorio está incluido dentro de las estrategias de enseñanza que extienden el concepto de aprendizaje activo a las distintas actividades del aula. RTP se desarrolla como trabajo de laboratorio y está diseñada para complementarse con una estructura tradicional de la materia, basada en clases expositivas y resolución de problemas, como así también, puede formar parte de una programación de aprendizaje activo que involucre otras metodologías de enseñanza. RTP utiliza recursos didácticos como conflicto y puentes cognitivos, a través de un ciclo de aprendizaje que consta de: exploración, introducción y aplicación de conceptos. Esta metodología pone al servicio del proceso de aprendizaje una moderna tecnología de información y comunicación con el objetivo de que los alumnos revean su interpretación del mundo físico en términos de válidos principios científicos (Sokoloff, D. et al., 2010).

El desarrollo de la serie de guías de laboratorio de RTP se inició en el año 1992 como parte de un esfuerzo continuo para crear materiales curriculares de alta calidad, instrumentos informáticos y sensores 
electrónicos para ayudar en la enseñanza de la física introductoria. El desarrollo de todos estos materiales curriculares se ha guiado por los resultados de la investigación en educación de la Física. Esta investigación nos ha llevado a creer que los estudiantes pueden aprender conceptos físicos de manera más efectiva a través de actividades guiadas que se han mejorado mediante el uso de potentes laboratorios (Sokoloff et al., 2004).

Para el presente estudio se utiliza el Laboratorio № 3: Voltaje en Circuitos de Corriente Continua Simples y Ley de Ohm que forma parte de la serie de laboratorios de RTP. En este laboratorio se exploran las distintas diferencias de potencial en circuitos serie y paralelo, que se construyen con lámparas y resistores. En el mismo laboratorio los alumnos deben intentar descifrar la Ley de Ohm estudiando la relación que existe entre la corriente a través de una resistencia y el voltaje presente en la misma (Sokoloff et al., 2004). Los temas a tener en cuenta para ser cuantificados en los análisis experimentales a través del Test DIRECT son: i) Analizar y reconocer circuitos eléctricos simples; ii) Reconocer conexiones de los elementos en un circuito; iii) Reconocer un circuito cerrado; iv) Analizar el concepto de resistencia en serie y en paralelo; v) Aplicar el concepto de diferencia de potencial y obtener la relación entre corriente eléctrica, diferencia de potencial y resistencia eléctrica (Ley de Ohm).

RTP se desarrolla en tres pasos sucesivos: una tarea de predicción, realizada individualmente por el alumno y que debe ser entregada al comienzo del laboratorio. El segundo paso se desarrolla en el laboratorio, con actividades experimentales realizadas por grupos de 304 alumnos. Este trabajo es complementado en el último paso por una tarea individual que los alumnos deben realizar fuera de las horas de clase y entregar en la siguiente semana. En todas estas actividades el objetivo central es el aprendizaje conceptual de la física, también desarrollando algunas habilidades experimentales relacionadas con un trabajo práctico de laboratorio (Sokoloff et al., 2010).

La guía preparativa (primer paso) se administra antes de realizar el laboratorio y consta de unas pocas preguntas de formato abierto referidas a circuitos que se desarrollaran luego en la experiencia. El objetivo de este pequeño cuestionario es, por un lado, informar al docente sobre la situación inicial de conocimiento de los alumnos para poder hacer hincapié en ciertos temas, y por otro lado, preparar al estudiante sobre los temas que trata el trabajo de laboratorio. Esta tarea les toma a los alumnos un tiempo aproximado de veinte minutos. Al realizar el laboratorio (segundo paso), el alumno debe responder algunas preguntas más concretas sobre lo que observa experimentalmente. De esta manera, se lo conduce al alumno a descubrir no solo el funcionamiento de un circuito, sino también a descubrir la ley que lo fundamenta. Para poder realizar cualquier análisis de los circuitos los alumnos utilizan diferentes instrumentos de medida, con lo cual se consigue que el alumno además de cumplir con las metas establecidas por la experiencia en sí, empiece a familiarizarse con diferentes instrumentos fundamentales para analizar el funcionamiento de cualquier circuito eléctrico simple.

En el tercer y último paso esta metodología ofrece la posibilidad de desarrollar una actividad práctica fuera de clase. Esta tarea resume los tópicos abordados por las actividades realizadas con anterioridad y es efectuada una vez concluido el laboratorio. Este último paso, y no menos importante, conforma lo que es la aplicación de conceptos, luego de haber cumplimentado los ciclos de exploración e introducción.

\section{Instrucción Tradicional grupo control}

La instrucción teórica se realiza respetando la metodología tradicional de la asignatura de años anteriores. Las clases expositivas son llevadas a cabo por el profesor responsable de la materia y consisten básicamente en explicaciones teóricas con ayuda de la pizarra y presentaciones en formato digital. Estas clases cuentan con las principales definiciones sobre el tema de Circuitos Eléctricos y la exposición de algún ejemplo de aplicación característico. Cabe destacar que los distintos grupos tanto el de Control como ambos Experimentales recibieron estas clases teóricas dos días a la semana con una carga horaria total de 4 horas.

Otra actividad conjunta por parte de los grupos Control y Experimental son las clases de resolución de problemas prácticos que los alumnos trabajan luego de cada clase teórica en pequeños grupos de trabajo y con la supervisión de los auxiliares de práctico de la asignatura. La clase práctica de problemas representa una carga horaria de 5 horas semanales.

La actividad que resulta diferente para los grupos es el laboratorio de circuitos eléctricos, mientras que los grupos experimentales realizaron RTP, el grupo control efectuó un laboratorio de circuitos eléctricos básicos tradicional de la materia. La metodología de este laboratorio difiere a RTP en el hecho de que los estudiantes no elaboran su propio conocimiento, sino que siguen la receta propuesta por el docente a cargo. Cada actividad del laboratorio esta descripta de manera de que ellos solo repiten los pasos según el mismo 
los guía, no los invoca a realizar predicciones y contrastes con sus ideas anteriores. Este tipo de metodología responde a lo que se llama enseñanza tradicional y es lo que con este trabajo se pretende modificar para el tema de circuitos eléctricos.

\section{INSTRUMENTO DE EVALUACIÓN "DIRECT”}

Con motivos de evaluar las condiciones iníciales de los alumnos y analizar la evolución de los mismos se utiliza el test Determining and Interpreting Resistive Electric Circuits Concept Test (DIRECT) (Engelhardt y Beichner, 2004), esta prueba es un test de respuestas de opción múltiple desarrollado en la Universidad de North Carolina por el grupo de investigación en enseñanza de la física liderado por el Prof. Robert Beichner. El uso generalizado de instrumentos de prueba, como el Force Concept Inventory (FCl) (Hestenes et al., 1992) y el Test of Understanding Graphs in Kinematics (TUG-K) (Beichner, 1994) han sido desarrollados científicamente para evaluar la comprensión conceptual del estudiante. La prueba DIRECT en particular se desarrolló para evaluar la comprensión del estudiante en una variedad de conceptos de circuitos eléctricos resistivos de corriente directa (DC).

Esta es una prueba diseñada para determinar e interpretar los modelos estudiantiles sobre conceptos de circuitos eléctricos resistivos. Es un test que contiene entre sus distractores una verdadera taxonomía de concepciones alternativas y dificultades características de aprendizaje sobre circuitos eléctricos. Con la ayuda de esta herramienta, analizando las respuestas erróneas de los estudiantes podemos conocer sus preconcepciones y la importancia que estas poseen (Engelhardt y Beichner, 2004).

DIRECT es una prueba de 29 preguntas de respuestas de opción múltiple, cada pregunta tiene cinco opciones de respuesta, con distractores que corresponden a las concepciones previas y dificultades de aprendizaje más características sobre circuitos eléctricos. Cada concepto en particular está contenido en más de un ítem, de manera que el docente puede verificar la consistencia interna de las respuestas de los estudiantes controlando la correlación de las respuestas de ítems con un mismo objetivo. Los 29 ítems de DIRECT se distribuyen en 11 objetivos particulares o específicos. Estos a su vez están agrupados en 5 objetivos integradores (Ver Anexo). En el caso particular de esta investigación, la metodología no cubre la totalidad de los objetivos cubiertos por el test, por lo tanto en los análisis de resultados se hace hincapié en aquellos ítems de DIRECT que han sido cubiertos por la experiencia práctica (Sokoloff, D. et al., 2010).

En el caso particular de nuestra experiencia el test DIRECT se utiliza tanto como prueba inicial (Pre-test) antes de la instrucción (tradicional y experimental) para obtener de esta manera las ideas previas o preconcepciones sobre el tema de circuitos eléctricos resistivos que los alumnos poseen al iniciar el curso. Analizando las respuestas del Pre-test se puede enfocar o direccionar la enseñanza a algún tema en particular, en caso de ser necesario. En segundo lugar, el mismo cuestionario DIRECT se utiliza como Posttest con el objetivo de calcular la ganancia o efectividad que resultó de la instrucción, tanto tradicional como experimental. Cabe aclarar que las 29 preguntas del Test de respuestas de opción múltiple (DIRECT) son las mismas tanto para el Pre-test como para el Post-test. Luego con los datos numéricos obtenidos de las respuestas de los estudiantes se puede obtener una idea general de aquellos conceptos que fueron mejor entendidos por los alumnos que recibieron instrucción mediante la metodología RTP y en cuales la instrucción no ha sido completamente eficaz.

\section{RESULTADOS}

El primer análisis se enfoca en los rendimientos obtenidos por los alumnos, o sea, la cantidad de respuestas correctas obtenidas en el test DIRECT. Estos rendimientos se analizan teniendo en cuenta la totalidad de las preguntas del test, aunque algunas de ellas no han sido cubiertas por la instrucción experimental. El segundo análisis, se concentra en determinar la ganancia normalizada g (Ecuación 1), la cual fue introducida por Hake y sirve para poder determinar la fracción de la máxima ganancia posible (Hake, 1998), en este caso particular si se consideran solo aquellas preguntas cubiertas por la instrucción.

$$
g=\frac{\text { (promedio clase postest-promedio clase pretest })}{(100-\text { promedio clase pretest })} \times 100 \%
$$

En la Ecuación 1, el numerador expresa la ganancia media absoluta lograda por la instrucción, mientras que el denominador es la ganancia máxima que pudo haberse logrado (la diferencia entre el resultado óptimo y la condición inicial). Con esta definición, $g$ representa la fracción de la ganancia posible obtenida en dicho curso. Es por lo tanto un parámetro intensivo e independiente del estado inicial de conocimientos del curso, lo cual permite la comparación de la efectividad de una metodología de instrucción en cursos de muy diferente estado inicial (Benegas y Zavala, 2013). 


\section{Rendimientos Generales por Pregunta.}

En la Figura 1 y en la Figura 2 se muestran los rendimientos promedio de los estudiantes para cada una de las 29 preguntas del test DIRECT; en la Figura 1 se observa el Pre-test y en la Figura 2 el Post-test respectivamente. En ambas figuras se han sombreado aquellas preguntas no abordadas por la instrucción experimental (Preguntas 1, 2, 5, 11, 14, 20, 22, 23, 24 y 25). En estas figuras se nota que aquellas preguntas no abordadas por la instrucción experimental (sombreadas) tienen un bajo rendimiento y además mantienen estos valores entre el Pre-test y el Post-test, por lo tanto no existe una variación entre una instancia y la otra. Por otro lado, aquellas preguntas si alcanzadas por la experiencia, tienen un mejor rendimiento en el Post-test (Fig. 2) respecto al Pre-test (Fig. 1), lo cual nos indica que la intervención a través del Laboratorio RTP tuvo una influencia positiva. Las últimas barras de ambas figuras muestran el valor promedio alcanzado por los grupos tanto en el Pre-test como Post-test, en el caso de la Figura 1 la similitud entre estos valores indican la equivalencia de grupos al comenzar la instrucción, algo que mencionamos al comenzar esta investigación.

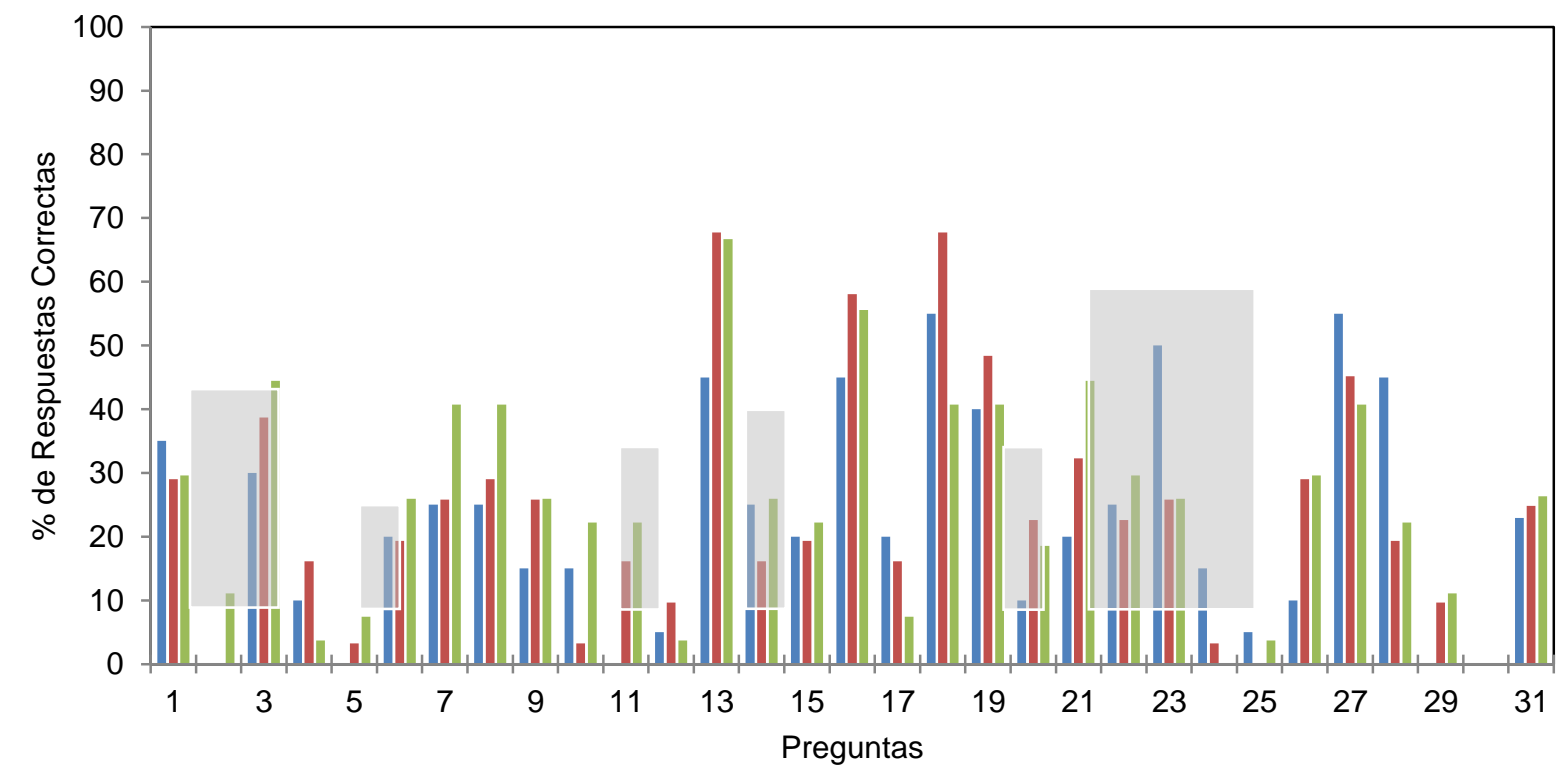

Fig. 1: Rendimiento promedio (\% respuestas correctas) Pre-Test para las muestras, "Control_A" (Barras azules) "Experimental_A" (Barras rojas) y "Experimental_B" (Barras verdes) para la totalidad de las preguntas de DIRECT.

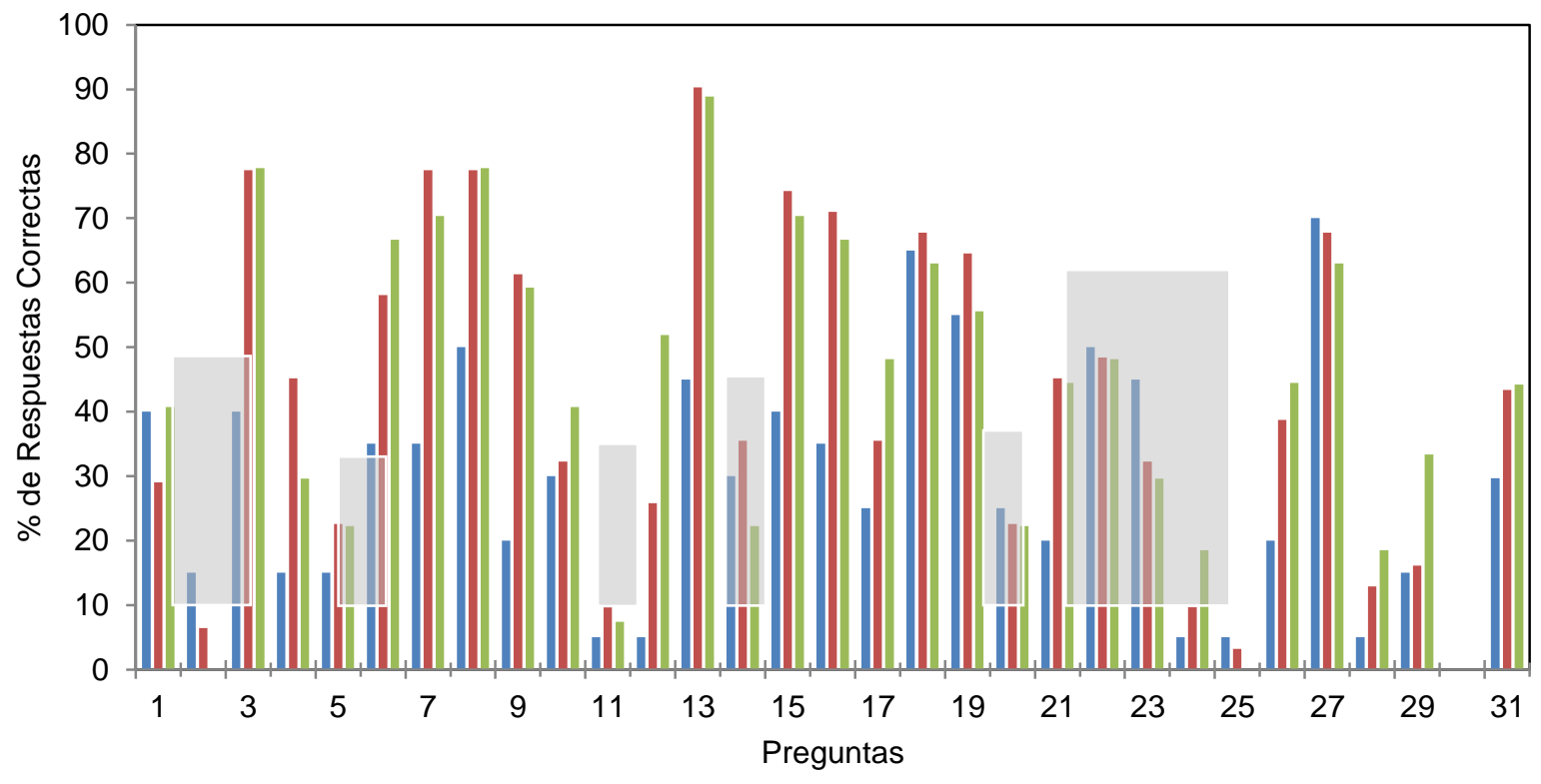

Fig. 2: Rendimiento promedio (\% respuestas correctas) Post-Test para las muestras, "Control_A" (Barras azules) "Experimental_A" (Barras rojas) y "Experimental_B" (Barras verdes) para la totalidad de las preguntas de DIRECT. 
El segundo análisis propuesto se observa en la Tabla 4, donde se muestran los resultados del Pre-test y Post-test de cada una de las muestras en estudio. En esta tabla también se observa la ganancia intrínseca, denominada coeficiente g (Hake, 1998), definido en la ecuación (1). Para los cálculos solo se han tenido en cuenta aquellas preguntas que fueron contenidas por la experiencia (Preguntas $3,4,6,7,8,9,10,12,13$, $15,16,17,18,19,21,26,27,28$ y 29 ).

Tabla 4: Promedios porcentuales de Pre Test y Post Test. Ganancia Intrínseca.

\begin{tabular}{|l|c|c|c|}
\hline Muestras & Pre-test & Post-test & $\mathrm{g}(\%)$ \\
\hline Control_A & $26,32 \pm 7,44$ & $32,89 \pm 8,34$ & $8,28 \pm 13,42$ \\
\hline Experimental_A & $30,56 \pm 9,74$ & $54,67 \pm 17,75$ & $35,14 \pm 22,31$ \\
\hline Experimental_B & $30,99 \pm 8,92$ & $56,34 \pm 15,98$ & $36,31 \pm 23,45$ \\
\hline
\end{tabular}

En la Tabla 4 se observan en la segunda y tercer columna las medias de las respuestas correctas obtenidas por cada grupo, tanto para el Pre-test como para el Post-test. Con estos valores se calcula a partir de la Ecuación 1 la ganancia normalizada que se muestra en la cuarta columna.

Con estos resultados se puede observar una mejora en la cantidad de respuestas correctas de los estudiantes desde el Pre-Test hacia el Post-test, acentuándose sobre todo en los grupos experimentales que recibieron la instrucción experimental propuesta. Esta diferencia se puede apreciar en las ganancias obtenidas por cada muestra, lo que demuestra el aporte de esta metodología de enseñanza aplicada conjuntamente con la enseñanza tradicional de la asignatura. Investigaciones anteriores muestran resultados similares sobre la aplicación de estas metodologías (Flores, 2008).

Tabla 5: Objetivos DIRECT

\begin{tabular}{|c|c|}
\hline Objetivos & İtems \\
\hline \multicolumn{2}{|l|}{ Aspectos físicos de circuitos eléctricos DC (Objetivos 1 a 5) } \\
\hline 1- Identificar y explicar un corto circuito (mas corriente sigue el camino de menor resistencia). & $\begin{array}{ll}10, & 19, \\
27 & \end{array}$ \\
\hline $\begin{array}{l}\text { 2- Comprensión de la conexión de elementos del circuito (los elementos tienen dos posibles puntos para } \\
\text { hacer una conexión). } \\
\text { 3- Identificar un circuito cerrado y entender la necesidad de un circuito cerrado para que la corriente pueda } \\
\text { fluir (no hay acumulación de cargas en ninguna parte del circuito). }\end{array}$ & 9,18 \\
\hline Combinación Objetivos 1 a 3 & 27 \\
\hline $\begin{array}{l}\text { 4- Aplicar el concepto de resistencia (obstáculo al flujo de cargas en un circuito) incluso esta resistencia } \\
\text { depende de la propiedad del objeto (geometría y tipo de material con que el objeto está compuesto). En un } \\
\text { circuito en serie la resistencia aumenta mientras más elementos se agregan, mientras que en paralelo a } \\
\text { medida que más elementos se agregan la resistencia disminuye. }\end{array}$ & $\begin{array}{ll}5, & 14, \\
23 & \end{array}$ \\
\hline $\begin{array}{l}\text { 5- Interpretación de dibujos y diagramas de una variedad de circuitos serie, paralelo y combinación de } \\
\text { ambos. }\end{array}$ & $\begin{array}{ll}4, & 13, \\
22 & \end{array}$ \\
\hline \multicolumn{2}{|l|}{ Esquema del circuito (Objetivos 1 a 3, 5) } \\
\hline \multicolumn{2}{|l|}{ Energía (Objetivos 6 a 7) } \\
\hline 6- Aplicación del concepto de potencia (trabajo por unidad de tiempo) a una variedad de circuitos. & 2,12 \\
\hline $\begin{array}{l}\text { 7- Aplicación de la comprensión conceptual de conservación de energía incluso la Ley de Kirchhoff ( } \Sigma \mathrm{V}=0 \\
\text { en una vuelta cerrada) y la batería como fuente de energía. }\end{array}$ & 3,21 \\
\hline \multicolumn{2}{|l|}{ Corriente (Objetivos 8 y 9) } \\
\hline 8- Entiendan y apliquen la conservación de corriente a una variedad de circuitos. & 8,17 \\
\hline $\begin{array}{l}\text { 9- Expliquen los aspectos microscópicos del flujo de la corriente en un circuito a través del empleo de } \\
\text { condiciones electrostáticas como el campo eléctrico, diferencias de potencial e interacción de fuerzas en } \\
\text { las partículas cargadas. }\end{array}$ & $\begin{array}{ll}1, & 11, \\
20\end{array}$ \\
\hline \multicolumn{2}{|l|}{ Diferencia de potencial (Voltaje) (Objetivos 10 y 11$)$} \\
\hline $\begin{array}{l}\text { 10- Aplicación del conocimiento de que la cantidad de corriente es influenciada por la diferencia de } \\
\text { potencial mantenido por la batería y la resistencia del circuito. }\end{array}$ & $\begin{array}{ll}7, & 16, \\
25 & \end{array}$ \\
\hline $\begin{array}{l}\text { 11- Aplicación del concepto de diferencia de potencial a una variedad de circuitos incluso el conocimiento } \\
\text { que la diferencia de potencial en un circuito serie es la suma, mientras que en un circuito en paralelo } \\
\text { permanece igual. }\end{array}$ & $\begin{array}{ll}6, & 15, \\
24, & 28, \\
29\end{array}$ \\
\hline Corriente y Voltaje (Objetivos 8 y 11) & 26 \\
\hline
\end{tabular}


El primer paso en el desarrollo de DIRECT fue un extenso y amplio examen de los libros de texto de secundaria y universidad, manuales de laboratorio, como así también discusiones informales con los instructores que utilizan estos materiales. Los objetivos que evalúa DIRECT se aprecian en la tabla 5, donde se observan los objetivos particulares como aquellos integradores, además se indican en la tabla los ítems que corresponden a cada objetivo (Engelhardt y Beichner, 2004).

\section{CONCLUSIONES}

A partir de los resultados obtenidos experimentalmente, de su análisis y de su discusión se pueden establecer las siguientes conclusiones de la aplicación de la metodología RTP: 1) con el uso de experimentos simples, como es el caso de un laboratorio de RTP, conjuntamente con la estructura tradicional de la materia, se consigue una mejora en el aprendizaje conceptual. Esto se ve reflejado en los mejores resultados conseguidos por los estudiantes; 2) es factible el uso de instrumentos de medición y evaluación, como lo es el test de respuestas de opción múltiple DIRECT, surgido a partir de diversos resultados de la investigación en enseñanza de la física; y 3) la aplicación conjunta de RTP y de instrumentos de medición como DIRECT proporciona una herramienta para planificar el dictado de las clases y a su vez una manera sencilla de evaluar los resultados obtenidos.

\section{REFERENCIAS}

Ausubel D., Novak J. y Hasenian H., "Psicología Educativa: Un punto de vista Cognoscitivo", Ed. Trillas, México (1976)

Beichner, R., “Testing student interpretation of kinematics graphs”, Am. J. of Physics, Vol. 62 (1994)

Benegas J., Villegas M., Pérez de Landazábal M. C., Otero J., "Conocimiento conceptual de física básica en ingresantes a carreras de ciencias e ingeniería en cinco universidades de España, Argentina y Chile”, Rev. lberoamericana de física, Vol. 5, №. 1, págs. 35-43 (2009)

Benegas J., Pérez de Landazábal M. C., Otero J., "El aprendizaje activo de la Física Básica Universitaria”, Capítulo 11, pp. 179-192, Ed. Andavira, España (2013)

Benitez Y., Mora C., "Enseñanza tradicional vs aprendizaje activo para alumnos de Ingeniería”, Rev. Cubana de Física, Vol. 27, No. 2A, pp.175-179, ISSN: 0253-9268 (2010)

Bono, R., “Diseños Experimentales: Prácticas”, Ed. Universitat de Barcelona, Barcelona (1997)

Calero, J., "Investigación Cualitativa y Cuantitativa: Problemas no resueltos en los debates actuales", Rev. Cubana Endocrinol 2000, 11 (3): 192-8 (2000)

Campbell, D., “Experimental and Quasi-Experimental Designs for Research”, Ed. Rand McNally, Chicago, Illinois (1963)

Campbell, D., Stanley J., "Diseños experimentales y cuasi experimentales en la investigación social”, Ammorrortu Editores, Buenos Aires, Argentina (2002)

Castorina, J., "Los problemas conceptuales del constructivismo y sus relaciones con la educación”, En Debates Constructivistas, Ed. Aiqué, Buenos Aires (1998)

Crowl, T., "Fundamentos de investigación educativa”, 2ª ed, Capítulo 14, pp. 183-203, Ed. McGraw-Hill, Boston, EEUU (2002)

Engelhardt, P. y Beichner, R., "Students understanding of direct current resistive electrical circuits", Am. J. of Physics, Vol. 72, pp. 98-115 (2004)

Flores, S., "Aprendizaje de Circuitos Eléctricos en el Nivel Polimodal: Resultado de distintas aproximaciones didácticas”, Revista Enseñanza de las Ciencias, 26 (2), pp. 245-246 (2008)

Hake, R., "Interactive-engagement versus traditional methods: A six-thousand-student survey of mechanics test data for introductory physics courses", Am. J. of Physics, Vol. 66, pp. 64-74 (1998)

Hestenes, D. et al., "Force Concept Inventory. The Physics Teacher", 141-58 (1992)

McDermott L., Redish E., "Resorce Letter: Per-1: Physics Education Research", American Journal of Physics, Vol. 67 (9) (1999) 
Mora C., "Fundamentos del aprendizaje activo de la Física", Memorias del V Congreso Internacional Didáctica de las Ciencias y X Taller Internacional sobre la Enseñanza de la Física, La Habana, Cuba (2008)

Regalado-Méndez A., Delgado-Vidal F., Martinez-Lopez R., Peralta-Reyes E., "Balanceo de Ecuaciones Químicas Integrando las Asignaturas de Química General, Algebra Lineal y Computación: Un Enfoque de Aprendizaje Activo", doi: 10.4067/S0718-50062014000200005, Rev. Formación Universitaria, Vol. 7(2), 29$40(2014)$

Sokoloff D., Thornton R. y Laws P., "Real Time Physics Module1: Mechanics, Module 2: Heat and Thermodynamics, Module 3: Electricity and Magnetism and Module 4: Light and Optics", $3^{\text {ra }}$ Edición, Ed. Wiley and Sons, EEUU (2012)

Sokoloff, D. et al., "Aprendizaje activo de la Física III, electricidad y magnetismo: manual de entrenamiento". Compilado por Benegas, J., $1^{\text {a }}$ Ed. San Luis, Argentina (2010) 